DIVISION OF THE HUMANITIES AND SOCIAL SCIENCES

CALIFORNIA INSTITUTE OF TECHNOLOGY

PASADENA, CALIFORNIA 91125

ALLOCATION RULES FOR NETWORK GAMES

Matthew O. Jackson

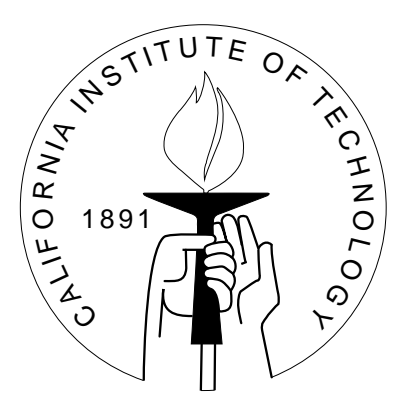

SOCIAL SCIENCE WORKING PAPER 1160 


\title{
Allocation Rules for Network Games
}

\author{
Matthew O. Jackson
}

\begin{abstract}
Previous allocation rules for network games, such as the Myerson Value, implicitly or explicitly take the network structure as fixed. In many situations, however, the network structure can be altered by players. This means that the value of alternative network structures (not just sub-networks) can and should influence the allocation of value among players on any given network structure. I present a family of allocation rules that incorporate information about alternative network structures when allocating value.
\end{abstract}

JEL classification numbers: C71, C72, A14

Key words: networks, core, Myerson Value, Shapley Value, allocation rules 


\title{
Allocation Rules for Network Games
}

\author{
Matthew O. Jackson*
}

\section{Introduction}

Consider a situation where a number of individuals or players are connected in some network relationship. The applications are quite wide and varied, ranging from friendships and social relationships, to communicating information about job openings, to business partnerships, to international trade agreements, and political alliances. What is in common to these situations is that the way in which players are connected to each other (for instance who talks to whom, or which countries have free-trade agreements) is important in determining the total productivity or value generated by the group. ${ }^{1}$

This paper examines the allocation of value among players connected by a network. How the fruits of the total productive value are allocated or transferred among players turns out to be important, not only in terms of fairness considerations, but also because it determines players' incentives to form various networks.

The allocation of value among a set of players has a rich axiomatic history in the cooperative game theory literature. There, the primitive information is what productive value is generated by each possible group of agents or coalition. Well-known rules such as the Shapley Value and Nucleolus provide natural ways of allocating the value among players. However, as mentioned above, in many applications the value generated by some group of players depends not only on their identities but also on how they are connected to each other. That is, alternative network structures (e.g., communication lines, alliances, friendships, etc.) connecting the same set of players might lead to very different costs and benefits. Thus, in many situations it will be important to account for network structure and not just coalition structure.

Myerson (1977) made a seminal contribution in adapting the cooperative game theory structure to accommodate information about the network connecting players. The way in which he did this was by augmenting a cooperative game by a network structure which

${ }^{*}$ I thank Jernej Copic, Sergio Currarini, and Gabrielle Demange for helpful discussions and comments on earlier drafts. Financial support from the Lee Center for Advanced Networking is gratefully acknowledged.

${ }^{1}$ See Jackson (2003) for a recent survey of some of the literature on network games. 
might be viewed as the lines of communication open to players. Once the network is fixed, such a communication game may be viewed as a cooperative game where the role of the network is to define which coalitions can function. The feasible coalitions are the ones whose members can freely communicate via the given network (so that any two players in the coalition are path connected in the network via players in the coalition). In a sense, each network structure and characteristic function (indicating how much value a given coalition can generate) induces a particular cooperative game. I will refer to this class as the class of communication games. ${ }^{2}$ Myerson (1977) showed that there exists a nice extension of the Shapley Value to communication games, and that it has a simple and intuitive characterization. This allocation rule has come to be called the Myerson Value in the subsequent literature. ${ }^{3}$

While such communication games are a useful augmentation of cooperative games, they fail to be rich enough to capture most applications where network structures are important. The shortcoming is easily seen. Consider a group of players $\{1,2,3\}$. Under this sort of communication game, this group generates the same productive value whether (i) they are each connected to each other via a complete network (a link between 1 and 2, a link between 2 and 3, and a link between 1 and 3); or (ii) they are connected via a less complete network such as one with a link between 1 and 2 and a link between 2 and 3, but no link between 1 and 3. To the extent that there are any costs to links, or benefits from shortened paths, etc., it will generally be the case that the value generated in these two scenarios differs.

Jackson and Wolinsky (1996) introduced a class of games where the value generated depends directly on the network structure. This allows the value generated by the three players $\{1,2,3\}$ to depend on the network structure so it can differ between cases (i) and (ii) above. I will refer to this class as the class of network games. Network games include cooperative games and communication games as special cases, but generally allow for costs and benefits to accrue differently to different sets of links, and allow for externalities and such across players and networks. Jackson and Wolinsky (1996) showed that the Myerson Value has a direct extension from communication games to network games.

While network games are richer than communication games and readily apply to a wide class of situations, the extension of the Myerson value to network games still inherits much of its perspective from communication games. Most importantly, under the Myerson value the network is implicitly viewed as fixed when value is being allocated. In particular, while the Myerson Value involves Shapley Value style calculations that account for how the network is built up, it does not account for the value of alternative network structures that might have formed. I shall return to illustrate and discuss this in more detail below.

Here, I take the view that the network is not a permanent fixture, but is something

\footnotetext{
${ }^{2}$ Myerson (1977) referred to the networks as cooperation structures. Much of the literature that followed Myerson has used the term communication structures, and so I will call those communication games.

${ }^{3}$ See Aumann and Myerson (1988).
} 
that is either being formed or might change in the future. From this point of view the allocation of value at a given network can and should depend on the value that might accrue to alternative potential networks. In particular, evaluating the contribution to value of a given link or player depends on the contribution of that link or player to various networks, and perhaps more importantly, which to what extent other links or players might serve as a substitute.

To understand why this issue arises in network games, but not in either cooperative games or communication games, it is important to recognize that in a cooperative game or communication game it is generally assumed that it is efficient for the grand coalition to form. ${ }^{4}$ The idea is that the grand coalition can always do whatever smaller coalitions can do, and possibly more. Once one moves to network games, however, larger networks might have higher costs associated with them than some smaller networks. For instance, the value generated by the complete network might be much less than the value generated by some sub-network network. This means that generally, the most efficient network (in a value maximizing sense) might not be the complete network. This introduces some important new considerations in the allocation of value that did not arise in the context of cooperative or communication games.

The considerations that are important in network games but did not arise in cooperative and communication games are most easily seen through some examples that I will present shortly. But to get some preview of what is going on, let me discuss it briefly now. In order to figure out how to allocate value across players, a rule such as the Shapley Value decomposes the grand coalition in various ways in evaluating players' contributions. However, given that the grand coalition is the ultimate one that forms, the issue of what other coalitions might have formed, or what other players might have played the role of a given player never really arise. In contrast, in the context of a network game, it is often (perhaps even generally) the case given any implicit or explicit costs to links that the set of efficient networks will not include the complete network. This means that we must care about how to allocate value to some networks that are not complete networks. In such cases, the allocation of value may depend on information about the roles of given players that involve calculations based on networks that are not subnetworks of a given network. For instance, if we consider a player at the center of a star network (where all players are connected to this center player but not directly to each other), how much value accrues to this player might depend on whether this player is the only one who can serve this function, or whether it is the case than any player could equally well have served as the center in a productive way.

The paper proceeds as follows. Section 2 provides definitions for network games. Section 3 provides an example illustrating some of the issues. Section 4 introduces two new allocation rules and characterizes them. Section 5 provides a definition of the core for network games and two other new allocation rules. Section 6 provides a general method of defining allocation rules. Section 7 concludes with a comparison of the properties

\footnotetext{
${ }^{4} \mathrm{An}$ exception are cooperative games in partition function form, as introduced by Lucas and Thrall (195?). However, that literature has not developed methods of allocating value that would help here.
} 
satisfied by various allocation rules.

\section{Network Games and Allocation Functions}

\section{Players}

$N=\{1, \ldots, n\}$ is a set of players who are connected in some network relationship.

The set $N$ will generally be fixed in what follows.

\section{Networks}

A network is a list of which pairs of players are linked to each other and is modeled as a non-directed graph. A network is thus a list of unordered pairs of players $\{i, j\}$, where $\{i, j\} \in g$ indicates that $i$ and $j$ are linked under the network $g$.

For simplicity, write $i j$ to represent the $\operatorname{link}\{i, j\}$, and so $i j \in g$ indicates that $i$ and $j$ are linked under the network $g$.

More formally, let $g^{N}$ be the set of all subsets of $N$ of size 2. $G=\left\{g \subset g^{N}\right\}$ denotes the set of all possible networks or graphs on $N$.

For instance, if $N=\{1,2,3\}$ then $g=\{12,23\}$ is the network where there is a link between players 1 and 2, a link between players 2 and 3, but no link between players 1 and 3 .

The network obtained by adding link $i j$ to an existing network $g$ is denoted $g+i j$ and the network obtained by deleting link $i j$ from an existing network $g$ is denoted $g-i j$.

Let $N(g)$ be the set of players who have at least one link in $g$. That is,

$$
N(g)=\{i \mid \exists j \text { s.t. } i j \in g\} .
$$

Let $n(g)=\# N(g)$ be the number of players involved in $g$.

Let $L_{i}(g)$ be the set of links that player $i$ is involved in, so that

$$
L_{i}(g)=\{i j \mid \exists j \text { s.t. } i j \in g\}
$$

and let $\ell_{i}(g)=\# L_{i}(g)$.

Let $\ell(g)=\sum_{i} \ell_{i}(g)$ be the number of links in $g$.

\section{Networks on Subsets of Players}


Given any $S \subset N$, let $g^{S}$ be the set of all subsets of $S$ of size 2, so that $g^{S}$ is the complete network among the players in $S$.

Let

$$
\left.g\right|_{S}=\{i j: i j \in g \text { and } i \in S, j \in S\} .
$$

Thus $\left.g\right|_{S}$ is the network found deleting all links except those that are between players in $S$.

Note the important distinction between the notation $g^{S}$ which is the complete network among players in $S$, and $\left.g\right|_{S}$ which is the network found by starting with some $g$ and then eliminating links involving players outside of $S$.

\section{Paths}

A path in a network $g \in G$ between players $i$ and $j$ is a sequence of players $i_{1}, \ldots, i_{K}$ such that $i_{k} i_{k+1} \in g$ for each $k \in\{1, \ldots, K-1\}$, with $i_{1}=i$ and $i_{K}=j$.

\section{Components}

Looking at the path relationships in a network naturally partitions a network into different connected subgraphs that are commonly referred to as components.

A component of a network $g$, is a nonempty subnetwork $g^{\prime} \subset g$, such that

- if $i \in N\left(g^{\prime}\right)$ and $j \in N\left(g^{\prime}\right)$ where $j \neq i$, then there exists a path in $g^{\prime}$ between $i$ and $j$, and

- if $i \in N\left(g^{\prime}\right)$ and $j \notin N\left(g^{\prime}\right)$ then there does not exist a path in $g$ between $i$ and $j$.

Thus, the components of a network are the distinct connected subgraphs of a network.

The set of components of $g$ is denoted $C(g)$. Note that $g=\cup_{g^{\prime} \in C(g)} g^{\prime}$.

Under this definition of component, a completely isolated player who has no links is not considered a component.

\section{Value Functions}

A value function is a function $v: G \rightarrow \mathbb{R}$.

The set of all possible value functions is denoted $V$.

A value function specifies the total value that is generated by a given network structure. The calculation of value may involve both costs and benefits and is a richer object than a characteristic function of a cooperative game, or that induced in a communication 
game, as it allows the value that accrues to depend on the network structure and not only on the coalition of players involved (or who can communicate).

An interesting sub-class of value functions are those where the value to a given component of a network does not depend on the structure of other components. This precludes externalities across (but not within) components of a network. While the characterizations below will apply more broadly, understanding behavior on component additive value functions is useful in characterizing the Myerson Value.

A value function $v$ is component additive if $v(g)=\sum_{g^{\prime} \in C(g)} v\left(g^{\prime}\right)$ for any $g \in G$ and $g^{\prime} \in C(g)$.

\section{Efficient Networks}

A network $g \in G$ is efficient relative to a value function $v$ if $v(g) \geq v\left(g^{\prime}\right)$ for all $g^{\prime} \in G$.

Thus, efficient networks are value-maximizing networks.

\section{Monotonic Covers}

When we begin to think about the possibilities available to a set of links when there is still some possibility of altering the network, it will useful to consider the value generated by the maximum over possible networks that could be formed using the given set of links links. This is captured by its monotonic cover.

Given a value function $v$, its monotonic cover $\widehat{v}$ is defined by

$$
\widehat{v}(g)=\max _{g^{\prime} \subset g} v\left(g^{\prime}\right) .
$$

\section{Monotonicity}

A value function $v$ is monotonic if $v\left(g^{\prime}\right) \geq v(g)$ whenever $g \subset g^{\prime}$.

Note that a value function is monotonic if and only if $v=\widehat{v}$.

In general the value functions that are natural in network games will not be monotonic. Nevertheless, information about how allocation rules perform on monotonic games will be useful.

\section{A Basis for Value Functions}

It will be useful to define a basis for the set of value functions. 
Let $v_{g}$ denote the value function that satisfies

$$
v_{g}\left(g^{\prime}\right)= \begin{cases}1 & \text { if } g \subset g^{\prime} \text { and } \\ 0 & \text { otherwise }\end{cases}
$$

Call such a $v_{g}$ a basic value function.

Note that any $v$ can be written as a linear combination of basic value functions $v_{g}$ 's. That is, for any $v$ we can write $v=\sum_{g} c_{g} v_{g}$ for some collection of scalars $c_{g}$. This follows from viewing the $v$ 's as vectors in $\mathbb{R}^{2^{n(n-1) / 2}}$ and noting that the $2^{n(n-1) / 2}$ different $v_{g}$ 's are linearly independent and so form a basis for $\mathbb{R}^{2^{n(n-1) / 2}}$.

\section{Network Games}

A network game is a pair, $(N, v)$, of a set of players and a value function.

\section{Allocation Rules}

How the value generated by a network is allocated among the players, either through their decisions or perhaps even by some outside intervention, is described by an allocation rule.

An allocation rule is a function $Y: G \times V \rightarrow \mathbb{R}^{n}$ such that $\sum_{i} Y_{i}(g, v)=v(g)$ for all $v$ and $g$.

Note that balance $\left(\sum_{i} Y_{i}(g, v)=v(g)\right)$ is built into the definition of an allocation rule.

It is important to note that an allocation rule depends on both $g$ and $v$. This allows an allocation rule to take full account of an player $i$ 's role in the network. This includes not only what the network configuration is, but also how the value generated depends on the overall network structure. For instance, consider a network $g=\{12,23\}$ in a situation where $v(g)=1$. Player 2's allocation might be very different depending on what the values of other networks are. For instance, if $v(\{12,23,13\})=0=v(\{13\})$, then in a sense 2 is essential to the network and may receive a large allocation. If on the other hand $v\left(g^{\prime}\right)=1$ for all networks, then 2's role is not particularly special. This information might turn out to be relevant, which is why the allocation rule is allowed (but not required) to depend on it.

\section{The Myerson Value and some Examples}

As mentioned in the introduction, Myerson (1977) developed a variation of the Shapley value for communication games, which was subsequently referred to as the Myerson 
Value. The Myerson Value also has a corresponding allocation rule in the context of network games which is a direct generalization of the version defined by Myerson for communication games, as shown by Jackson and Wolinsky (1996). That allocation rule is expressed as follows.

$$
Y_{i}^{M V}(g, v)=\sum_{S \subset N \backslash\{i\}}\left(v\left(\left.g\right|_{S \cup i}\right)-v\left(\left.g\right|_{S}\right)\right)\left(\frac{\# S !(n-\# S-1) !}{n !}\right)
$$

The Myerson value is characterized by the following properties.

\section{Component Balance}

An allocation rule $Y$ is component balanced if for any component additive $v, g \in G$, and $g^{\prime} \in C(g)$

$$
\sum_{i \in N\left(g^{\prime}\right)} Y_{i}(g, v)=v\left(g^{\prime}\right)
$$

Note that component balance only makes requirements on $Y$ for $v$ 's that are component additive, and $Y$ can be arbitrary otherwise. If $v$ is not component additive, then requiring component balance of an allocation rule $Y(\cdot, v)$ would necessarily violate balance.

Component balance requires that if a value function is component additive, then the value generated by any component to be allocated to the players among that component.

\section{Equal Bargaining Power}

An allocation rule satisfies equal bargaining power ${ }^{5}$ if for any component additive $v$ and $g \in G$

$$
Y_{i}(g, v)-Y_{i}(g-i j, v)=Y_{j}(g, v)-Y_{j}(g-i j, v) .
$$

Note that equal bargaining power does not require that players split the marginal value of a link. It just requires that they equally benefit or suffer from its addition. It is possible (and generally the case) that $Y_{i}(g)-Y_{i}(g-i j)+Y_{j}(g)-Y_{j}(g-i j) \neq v(g)-v(g-i j)$.

\section{A Characterization of the Myerson Value}

The following characterization of the Myerson value is from Jackson and Wolinsky (1996) and is an easy extension of a theorem of Myerson (1977). ${ }^{6}$

\footnotetext{
${ }^{5}$ This was called "fairness" by Myerson (1977).

${ }^{6}$ Dutta and Mutuswami (1997) extend the characterization to allow for weighted bargaining power, and show that one obtains a version of a weighted Myerson Value.
} 
Theorem $1 Y$ satisfies component balance and equal bargaining power if and only if $Y(g, v)=Y^{M V}(g, v)$ for all $g \in G$ and any component additive $v$.

Note that the above characterization only holds on component additive value functions. $^{7}$

\section{Examples and Criticisms}

Let us now examine a series of examples that I argue show shortcomings of the Myerson Value in network games, and also provide insight into how those shortcomings might be traced to the conditions of equal bargaining power and component balance.

Example 2 Insensitivity of the Myerson Value to Alternative Networks

Let $v(\{12\})=v(\{23\})=1, v(\{12,23\})=1$, and $v(g)=0$ for all other networks. 1.

Let $v^{\prime}(g)=1$ for all $g \neq \emptyset$. That is, under $v^{\prime}$ the value of any non-empty network is

So, under $v$ player 2 is need to generate any value, while under $v^{\prime}$ all non-empty networks generate the same value and so player 2's criticality is quite different under the two value functions.

Note, however, that the Myerson Value is insensitive to this information, as for instance

$$
Y^{M V}(\{12,23\}, v)=Y^{M V}\left(\{12,23\}, v^{\prime}\right)=\left(\frac{1}{6}, \frac{2}{3}, \frac{1}{6}\right) .
$$

\footnotetext{
${ }^{7}$ Myerson (1977) built component balance into his definition of an allocation rule, as the value that accrues to the grand coalition in a communication game is by definition determined in a component additive way. However, once one turns to network games one admits the possibility of externalities across components and has to be explicit about the component additivity of the value function in order to extend Myerson's result.
} 


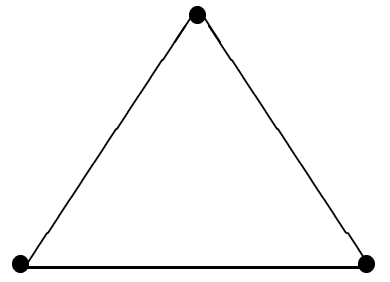

$$
\mathrm{v}=0, \mathrm{v}^{\prime}=1
$$

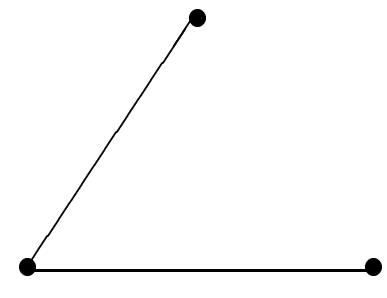

$\mathrm{v}=0, \mathrm{v}^{\prime}=1$

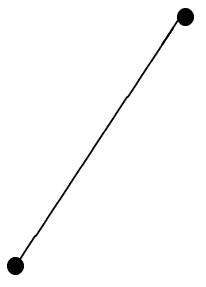

$\mathrm{v}=\mathrm{v}^{\prime}=1$

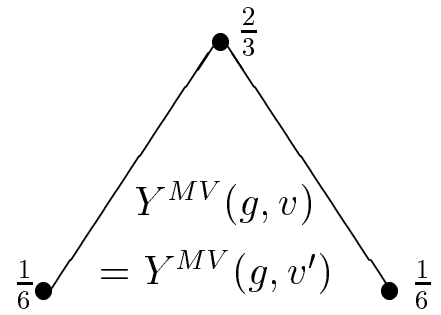

$\mathrm{v}=\mathrm{v}^{\prime}=1$

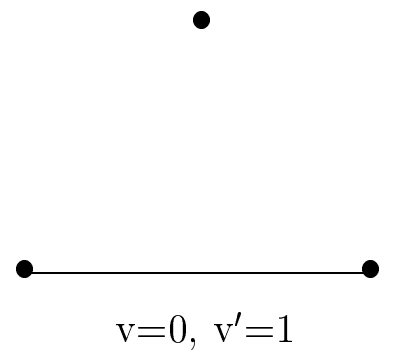

.

$\mathrm{v}=\mathrm{v}^{\prime}=0$

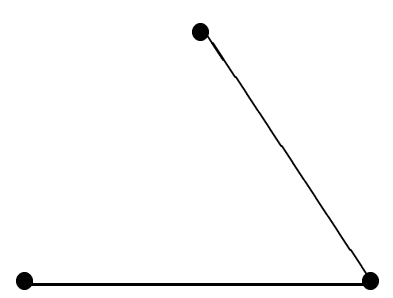

$\mathrm{v}=0, \mathrm{v}^{\prime}=1$

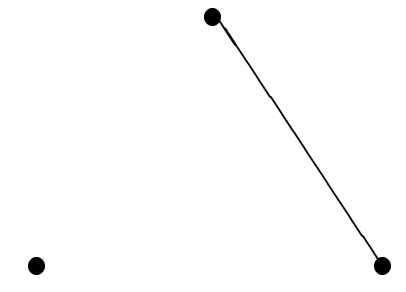

$\mathrm{v}=\mathrm{v}^{\prime}=1$

Figure 1

Here, regardless of the distinction between $v$ and $v^{\prime}$, player 2 gets a larger allocation in the network $\{12,23\}$ than the other players. This reflects player 2's status in two links in the network, and comes about through the Shapley value style calculations underlying the Myerson value, where we can think of building up the network $\{12,23\}$ by adding players one at a time. 
While player 2's role is special in the network $\{12,23\}$ in the sense that she is involved in two links while the other players are only involved in one, player 2's status is not special under the value function $v^{\prime}$ in the sense that any player could serve the same role. If the allocation of value is decided upon based at a stage where the network is not completely permanent, ${ }^{8}$ the fact that any player can equally well serve the central role, should have an impact on player 2's allocation. In fact, it seems natural that under $v^{\prime}$ all players should receive equal payments.

The insensitivity of the Myerson value to differences in value functions such as the difference between $v$ and $v^{\prime}$ is the motivation for this paper, and I propose alternative allocation rules that will account for such variations in value functions.

Before proceeding, let us examine some examples that provide a look at problems with the axioms underlying the Myerson value. This will help us in understanding the issues more deeply and in developing alternative allocation rules.

Example 3 A Criticism of Equal Bargaining Power

Let $v(\{12\})=v(\{23\})=1$ and $v(g)=0$ for all other networks.

Other networks have $v=0$
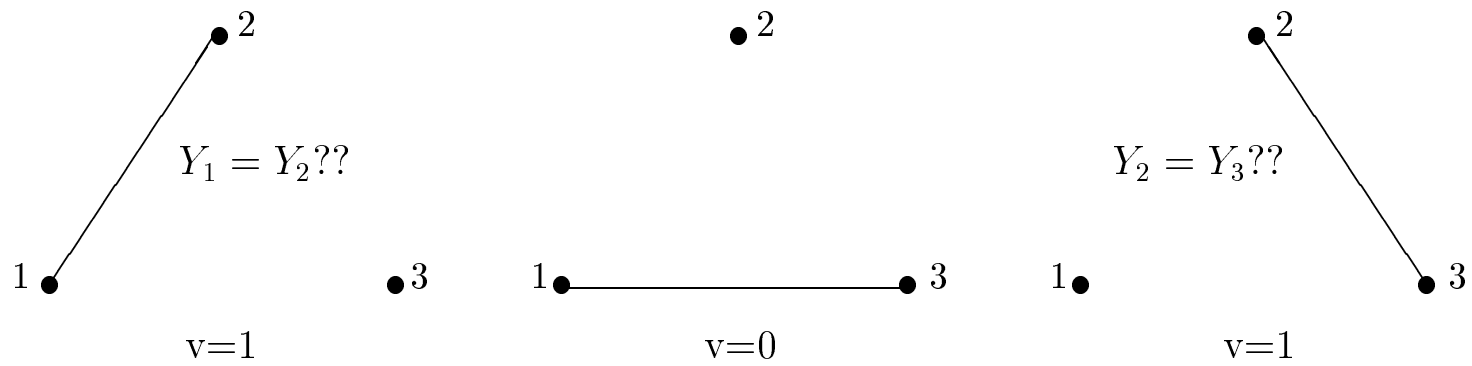

Figure 2

Any allocation rule, including the Myerson value, that satisfies equal bargaining power (and allocates 0 to the players on the empty network) will satisfy $Y_{1}(\{12\}, v)=$ $Y_{2}(\{12\}, v)$.

Here, there is a real asymmetry among the players and player 2 is more a critical player than the others. It is not at all clear why we should require that the allocation to players 1 and 2 be the same in the network $\{12\}$, as player 2 has a viable outside option while player 1 does not.

\footnotetext{
${ }^{8}$ Even if the network is fixed, the full symmetry of players under $v^{\prime}$ suggests that we should treat them equally in allocating value.
} 
If we do want to impose some sort of fairness or equality in bargaining power, it should only apply when the roles of the players in question are really comparable. There is a clear sense in which player 2's bargaining power should be greater than that of the other players, and so the title of "equal bargaining power" is in a sense a misnomer.

As a further note, consider $v^{\prime}$ such that $v^{\prime}(\{12\})=v^{\prime}(\{23\})=v^{\prime}(\{13\})=1$ and $v(g)=0$ for all other networks.

Note that here again, equal bargaining power imposes the two agents having a link in a one-link network each get an allocation of $\frac{1}{2}$. In fact, component balance and any sort of anonymity condition imply the same conclusion. However, under $v^{\prime}$ the full symmetry of agents suggests that the more natural allocation is $\frac{1}{3}$ to each player - including the player who is disconnected. This points to some of the difficulties with component balance. This is more fully illustrated in the following example.

Example 4 A Look at Component Balance

Let $v(g)=\frac{5}{4}$ if $g$ is a one-link network, $v(g)=2$ if if $g$ is a two-link network, and $v(g)=0$ otherwise.

In this case, the Myerson Value allocates $\frac{5}{8}$ to each player involved in the link in a one link network; and $\frac{1}{2}$ to players having one link in a two link network and 1 to the player having two links in a two link network.

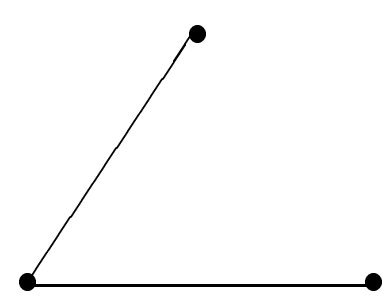

$\mathrm{v}=2$

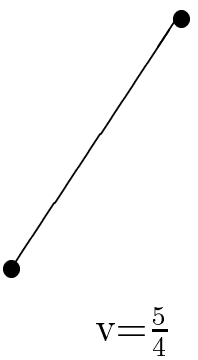

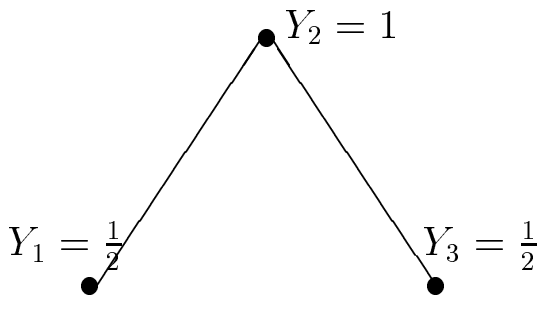

$\mathrm{v}=2$

$\downarrow$

$Y_{2}=0$

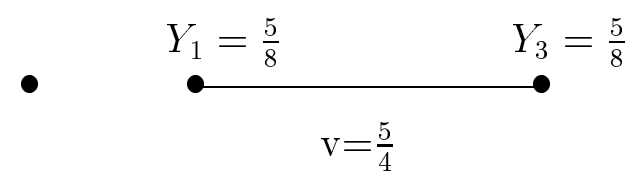

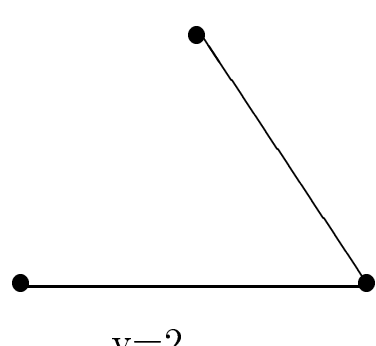

$\mathrm{v}=2$

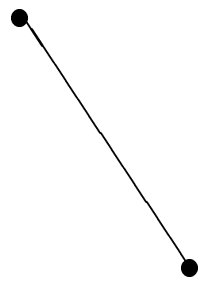

$\mathrm{v}=\frac{5}{4}$ 
Figure 3

Why might one criticize component balance here? It is based on noting that component balance sits in a sort of "no-man's-land" here: it is not quite strong enough to capture its normative arguments, but at the same time it is strong enough to rule out other natural conditions.

To be more explicit, a natural and reasonable argument (and the best I can think of) behind component balance is that it makes sure that components of a network receive their due value which prevents their members from wanting to walk away and reallocate their value among themselves. That is, in the absence of component balance, some component is necessarily receiving less than the value it is generating. If that component can choose to walk away and operate on their own, then they would benefit by doing so, which will upset the allocation rule. ${ }^{9}$

However, if one uses this type of argument to justify component balance, then it is logical to worry about all coalitional deviations, not just those of components. This means that the natural logical conclusion of the argument motivating component balance should actually be a core property. Note however, that component balance does not guarantee that an allocation lies in the core (for a formal definition see Section 5). For example, under the Myerson value above, if the network is $\{12,23\}$, then players 1 and 3 receive $\frac{1}{2}$ each, and would benefit by deviating and forming their own one link network where they could receive $\frac{5}{8}$ each.

On the other hand, while component balance stops short of dealing with all relevant coalitional possibilities, it is already strong enough to be in conflict with some fairness and anonymity properties. In the above example, the $v$ is completely anonymous and all players are completely interchangeable. If we then think of the value being bargained upon at a stage where the network is still flexible, it is not clear why value is not split equally among the players regardless of which of which network is formed. This strong form of anonymity is precluded by component balance.

The criticisms of the two properties lie on different levels. The criticism of equal bargaining power is that it is a fundamentally flawed property. The criticism of component balance is that it is not strong enough to reflect its real normative grounding, and yet strong enough to conflict with other properties. Thus, one might prefer to either strengthen component balance if one really is concerned with what value is generated by various coalitions and components, or abandon the property if one is more concerned with other normative properties.

I suggest alternative approaches below. Both involve weakening the flawed equal bargaining power to only apply in situations where players are equal in some broader sense.

\footnotetext{
${ }^{9}$ Remember that component balance is always operating in the context of component additive value functions, and so such walking away can occur without worry about what the remaining agents do.
} 
The difference in the two approaches depends on whether (i) one replaces component balance with some anonymity or equity properties, or (ii) strengthens component balance to be a core property. Depending on the variation, one will end up with very different allocation rules.

\section{Flexible Networks and Equal Treatment}

In this section I provide two new allocation rules which are based on eliminating equal bargaining power, and working with equity properties in place of component balance. The two variations depend on whether one assesses value on a player by player basis, or on a link by link basis.

It is not clear to me that either variation is clearly more pertinent than the other. In one sense the link-based rule is richer in that it considers variations on a link-by-link basis, and presumably players could choose to withhold certain links and not others. On the other hand, it is really the players who control the links and not the links themselves who accumulate value (as becomes clearer when dealing with a core definition). I first present the player-based rule and then the link-based version.

Before providing the allocation rules, let us consider some conditions important in characterizing them.

The first condition is one that captures the basic idea that the allocation of value is taking place with the perspective that the network is something that can be varied.

\section{Flexible-Network Rules}

An allocation rule $Y$ is a flexible network rule if $Y(g, v)=Y\left(g^{N}, \widehat{v}\right)$ for all $v$ and efficient $g$ (relative to $v$ ).

The idea that the allocation only depends on the monotonic cover of the value function is one property that is implied from the perspective that the allocation is being decided upon when the network is formed or can still be changed: at a time where there is still some flexibility in the network. With the idea that inefficient networks should not be formed, the allocation of value should only depend on the value of efficient networks given some set of available links, and so the monotonic cover is all that enters into the calculations.

Note that this equivalence is only required on efficient networks, as the value on other

networks might not even be the same (i.e., $v(g) \neq \widehat{v}(g))$ and so the condition would be impossible to impose on inefficient networks. 
The next condition is a well-known foundation for Shapley-style calculations.

\section{Additivity}

An allocation rule $Y$ is additive if for any $v$ and $v^{\prime}$, and scalars $a \geq 0$ and $b \geq 0$

$$
Y\left(g^{N}, a v+b v^{\prime}\right)=a Y\left(g^{N}, v\right)+b Y\left(g^{N}, v^{\prime}\right) .
$$

In a way, one can think of additivity as a consistency or decomposition condition. The way in which value is allocated may be broken down so that one may separately allocate the value on different parts of the value function and then sum up. This requires that same logic is imposed on each part of the value function.

In the context of network games is a bit too strong. It may be in conflict with making decisions from a flexible network perspective. In particular, the monotonic cover of $v+v^{\prime}$ is not necessarily the same as the sum of the monotonic covers.

This suggests a more limited version of additivity.

\section{Weak Additivity}

An allocation rule $Y$ is weakly additive if for any monotonic $v$ and $v^{\prime}$, and scalars $a \geq 0$ and $b \geq 0$

$$
Y\left(g^{N}, a v+b v^{\prime}\right)=a Y\left(g^{N}, v\right)+b Y\left(g^{N}, v^{\prime}\right) .
$$

and if $a v-b v^{\prime}$ is monotonic, then

$$
Y\left(g^{N}, a v-b v^{\prime}\right)=a Y\left(g^{N}, v\right)-b Y\left(g^{N}, v^{\prime}\right) .
$$

The weaker version of additivity is one that is adapted to hold together with the flexible network perspective as it only applies in monotonic cases. While the value functions that we are interested in will rarely be monotonic, this condition will still be useful as we can apply the condition to monotonic covers.

The reasoning behind (weak) additivity is that if we are making calculations regarding allocations based on what players or links contribute to various network possibilities, and we increase or decrease those contributions, we should treat those increases or decreases in the same way that we treat the original contributions.

The key equity condition that I impose is the following.

\section{Equal Treatment of Vital Players}


An allocation rule $Y$ satisfies equal treatment of vital players if $v_{g}$ is a basic value function for some $g$, then

$$
Y_{i}\left(g, v_{g}\right)= \begin{cases}\frac{1}{n(g)} & \text { if } i \in N(g), \text { and } \\ 0 & \text { otherwise. }\end{cases}
$$

Recall that basic value functions are ones where the players involved in $g$ are all vital to the functioning of any network, in the sense that no value is generated without all the players in $g$ being present and that no other players contribute in any way.

In such a setting, the players in $g$ are all in some sense "equals" and all others contribute nothing. In this case, the equal treatment property allocates value equally to each player in $N(g)$.

This is a very weak equity condition and one that is satisfied by most allocation rules, with the exception of the egalitarian rules defined in Section 7.

\section{The Player-Based Flexible Network Allocation Rule}

First, let us define a rule for allocating value on efficient networks, and then we will come back to inefficient ones.

Consider any $v$ (not necessarily component additive) and a $g \in G$ that is efficient relative to $v$. Let

$$
Y_{i}^{P B F N}(g, v)=\sum_{S \subset N \backslash\{i\}}\left(\widehat{v}\left(g^{S \cup i}\right)-\widehat{v}\left(g^{S}\right)\right)\left(\frac{\# S !(n-\# S-1) !}{n !}\right)
$$

On a superficial level this rule bears some similarities to the Myerson Value because we see Shapley Value-like calculations. However, it is a quite different allocation rule. In fact, it violates both equal bargaining power and component balance, and is characterized by conditions that are violated by the Myerson Value. Most importantly it is a flexible network rule and thus accounts for the value of alternative possible networks. This is seen in its flexible network perspective, as it is relative to the monotonic cover $\widehat{v}$.

Theorem 5 An allocation rule satisfies equal treatment of vital players, weak additivity, and is a flexible network rule if and only if it is defined by (2) for all $v$ and $g$ that are efficient relative to $v$.

\section{Proportionality}

In order to have a full definition of an allocation rule, one needs also to define it for inefficient networks as well as efficient ones. When one takes a flexible network 
perspective, the question of how value is allocated on inefficient networks becomes a bit tricky, as it is not clear why inefficient networks should ever form. Nonetheless, we know that there exist some conflicts between stability and efficiency (e.g., see Jackson and Wolinsky (1996)). Thus, it is important to have something to say about the allocation of value on inefficient networks, both because such networks might end up forming, and also because their allocations might end up being important "off-the-equilibrium-path" considerations.

Here, I take the perspective that the relative allocations are the same on efficient and inefficient networks, so that the allocation is rescaled.

An allocation rule $Y$ is proportional if for each $i$ and $v$ either $Y_{i}(g, v)=0$ for all $g$, or for any $g$ and $g^{\prime}$ such that $v\left(g^{\prime}\right) \neq=0$

$$
\frac{Y_{i}(g, v)}{Y_{i}\left(g^{\prime}, v\right)}=\frac{v(g)}{v\left(g^{\prime}\right)}
$$

The proportionality condition takes an obvious (but certainly not the only natural) path to allocation of value on inefficient networks. Given the ex ante perspective, players allocations can be determined on efficient networks and then rescaled for inefficient ones. One might think of this as a way of saying that the efficient allocations have taken into account all of the relevant bargaining and decision making that would go on in terms of determining players' relative contributions or power due to a given value function.

This leads to the following extension of (2).

$$
Y_{i}^{P B F N}(g, v)=\frac{v(g)}{\widehat{v}\left(g^{N}\right)} \sum_{S \subset N \backslash\{i\}}\left(\widehat{v}\left(g^{S \cup i}\right)-\widehat{v}\left(g^{S}\right)\right)\left(\frac{\# S !(n-\# S-1) !}{n !}\right)
$$

Theorem 6 An allocation rule satisfies equal treatment of vital players, weak additivity, and is a flexible network and proportional rule if and only if it is $Y^{P B F N}$ (as defined in (3)).

Now let us turn to link-based allocation rules.

\section{Link-Based Allocation Rules}

An allocation rule $Y$ is link-based if there exists $\psi: V \times G \rightarrow \mathbb{R}^{n(n-1) / 2}$ such that $\sum_{i j \in g^{N}} \psi_{i j}(g, v)=v(g)$, and

$$
Y_{i}(g, v)=\sum_{j \neq i} \frac{\psi_{i j}(g, v)}{2}
$$


When thinking about how we measure players' contributions or how they bargain over their worth, this may be done directly in terms of adding or removing the players, or it may be done in terms of the links that they control. That is, we may think of assigning allocations to links and then this indirectly determines how value accrues to players.

The perspective of assigning values to links rather than players was first taken by Meeson (1988) (see also Borm, Owen, and Tijs (1992)) in the context of communication games and resulted in a variation on the Myerson Value called the Position Value (see Slikker (2000) for a discussion). More generally, we may think of the alternatives of doing player based or link based calculations when deriving any allocation rule in the context of network games as well.

\section{Equal Treatment of Vital Links}

An allocation rule $Y$ satisfies equal treatment of vital links if $v_{g}$ is a basic value function for some $g \neq \emptyset$, then

$$
Y_{i}\left(g, v_{g}\right)=\frac{\ell_{i}(g)}{2 \ell(g)}
$$

This property is a variation on equal treatment of vital players, where it is links who are viewed as being vital rather than players, and will correspond to a link-based version of an allocation rule.

This also might be thought of as reflecting the idea that it is not really the players that are vital, but certain collections of links (i.e., those in $g$ ). As such from one perspective it is the links which are all equal.

\section{The Link-Based Flexible Network Allocation Rule}

$$
Y_{i}^{L B F N}(g, v)=\frac{v(g)}{\widehat{v}\left(g^{N}\right)} \sum_{j \neq i}\left[\sum_{g \subset g^{N}-i j} \frac{1}{2}(\widehat{v}(g+i j)-\widehat{v}(g))\left(\frac{\# g !([n(n-1) / 2]-\# g-1) !}{[n(n-1) / 2] !}\right)\right] .
$$

To see how this rule works, proceed as follows. First, one may think of value being allocated to links, and so the allocation to a given player $i$ is simply that value summed across links. Note that value is allocated to a given link whether or not it is present in a given network - which reflects the flexible network perspective. The allocation on a given network is then rescaled in proportion to the value that would be allocated on an efficient network. The $\frac{1}{2}$ reflects the fact that the value of a given link is controlled by two players. The remaining part of the calculation is then based on a Shapley Value allocation of value to links as to what they contribute to the overall value possible under $v$. This calculation is from a flexible network perspective, so it is relative to the monotonic cover $\widehat{v}$. 
Theorem 7 An allocation rule satisfies equal treatment of vital links, weak additivity, and is an flexible network rule if and only if it agrees with $Y^{L B F N}$ on efficient networks. It satisfies equal treatment of vital links, weak additivity, and is an flexible network and proporitional rule if and only if it is $Y^{L B F N}$.

Again, note that the above characterization holds for all value functions, not just component additive ones.

Let us now compare the allocation of value by these flexible network bargaining rules to that of the Myerson Value.

Example 8 Example 2 Revisited

This is a slight generalization of Example 2.

Consider value functions $v$ and $v^{\prime}$ are defined as follows.

$v(\{12\})=v(\{23\})=1, v(\{12,23\})=w \geq 1$, and $v(g)=0$ for all other networks.

$v^{\prime}(g)=w$ for all $g$ with at least two links and $v^{\prime}(g)=1$ on $g$ with one link.

The Myerson Value allocates as follows.

$$
Y^{M V}(\{12,23\}, v)=Y^{M V}\left(\{12,23\}, v^{\prime}\right)=\left(\frac{w}{3}-\frac{1}{6}, \frac{w}{3}+\frac{1}{3}, \frac{w}{3}-\frac{1}{6}\right) .
$$

Here the link- and player-based flexible network allocation rules provide different allocations depending on values of networks that are not subnetworks of $\{12,23\}$.

$$
\begin{gathered}
Y^{L B F N}(\{12,23\}, v)=\left(\frac{w}{4}, \frac{w}{2}, \frac{w}{4}\right) . \\
Y^{P B F N}(\{12,23\}, v)=\left(\frac{w}{3}-\frac{1}{6}, \frac{w}{3}+\frac{1}{3}, \frac{w}{3}-\frac{1}{6}\right) . \\
Y^{L B F N}\left(\{12,23\}, v^{\prime}\right)=\left(\frac{w}{3}, \frac{w}{3}, \frac{w}{3}\right) . \\
Y^{P B F N}\left(\{12,23\}, v^{\prime}\right)=\left(\frac{w}{3}, \frac{w}{3}, \frac{w}{3}\right) .
\end{gathered}
$$



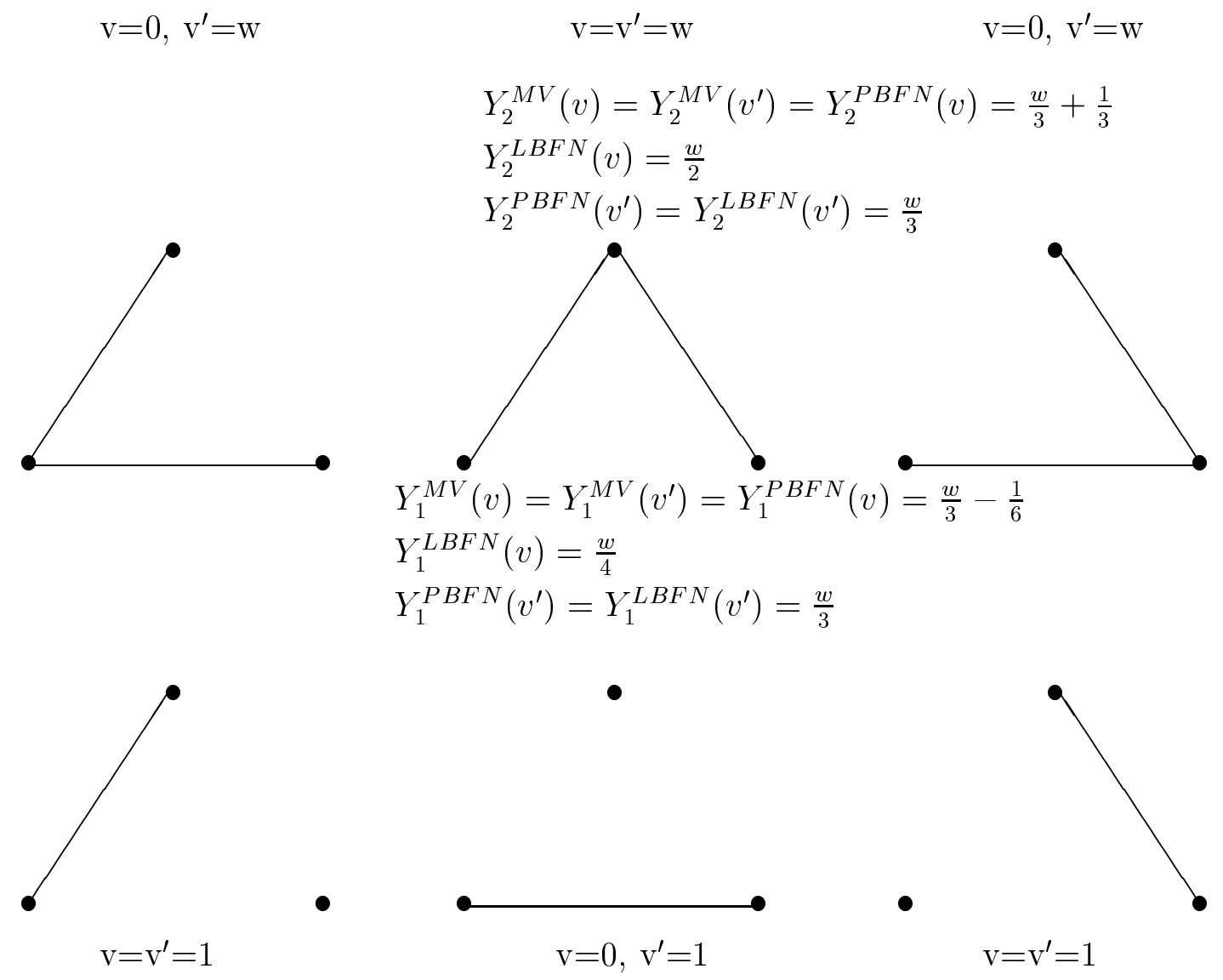

Figure 4

There are several things to note. Both allocation rules split values equally under $v^{\prime}$ which is consistent with the flexible network perspective and the equity conditions that they both satisfy.

The player-based allocation rule coincides with the Myerson Value on $v$ since under $v$ only subnetworks of $\{12,23\}$ generate any value. This differs from the link-based version. The link-based flexible network rule is easy to see here as only the links $\{12\}$ and $\{23\}$ generate value, but not the link $\{13\}$, and so the links split the value equally on $\{12,23\}$. Player 2 being involved in twice as many links as the others ends up with twice the value.

\section{The Core of a Network Game and the Networkolus}

While the flexible network allocation rules proposed in the previous section have nice properties in terms of their flexible network perspective, there is a shortcoming that they inherit from their Shapley Value origins. Namely, they are not always in the core of a network game. In situations where players are deciding on the allocation of the value 
of a network at the same time that they are forming the network there is a natural core definition that captures some constraints on the allocation of value that would be required to avoid certain forms of instability.

Requiring that an allocation rule be core consistent can be thought of as a strengthening of the component balance condition.

\section{The Core of a Network Game}

A network-allocation pair $g \subset g^{N}$ and $y \in \mathbb{R}^{n}$ is in the core of the network game $(N, v)$ if $\sum_{i} y_{i} \leq v(g)$ and $\sum_{i \in S} y_{i} \geq \widehat{v}\left(g^{S}\right)$ for all $S \subset N$.

The core of a network game provides a natural look at how the allocation of value of a network can be taken together with the formation of a network, especially regarding accounting for coalitional incentives.

Most of the literature on network games takes the allocation rule as given and then examines some non-cooperative formation procedure. ${ }^{10}$ There are some papers that have analyzed the allocation of value at the same time as the formation of a network. ${ }^{11}$ Those papers examine specific demand-based noncooperative network formation games. The core provides a protocol-free way analyzing the simultaneous allocation of value and formation of a network.

\section{Core Consistency}

An allocation rule $Y$ is core consistent, if for any $v$ such that the core is nonempty, there exists at least one $g$ such that $(g, Y(g, v))$ is in the core.

\section{Example 9 The Core}

Consider the value function $v$ from Example 8 except allowing $w$ to be any number, defined by $v(\{12\})=v(\{23\})=1, v(\{12,23\})=w$, and $v(g)=0$ for all other networks.

Note that the core is always non-empty, regardless of the value of $w$. If $w \leq 1$, then the networks $\{12\}$ and $\{23\}$ together with an allocation of $(0,1,0)$ are in the core. If $w>1$, then the network $\{12,23\}$ together with any allocations such that $y_{1}+y_{2} \geq 1$ and $y_{2}+y_{3} \geq 1$ are in the core. For instance, the allocation $\left(\frac{w-1}{2}, 1, \frac{w-1}{2}\right)$ is in the core together with the network $\{12,23\}$.

\footnotetext{
${ }^{10}$ For instance, see Jackson (2003) for a discussion of network formation games, as well as Goyal (2003) for discussion of dynamics and Page (2003) for discussion of farsightness.

${ }^{11}$ For instance, see Slikker and van den Nouweland (2000), Currarini and Morelli (2000), and Mutuswami and Winter (2000).
} 
Note, however, that when $w=\frac{7}{6}$, then the allocations of the Myerson, Player-Based Flexible Network, and Link-Based Flexible Network allocation rules are not in the core and thus fail core consistency.

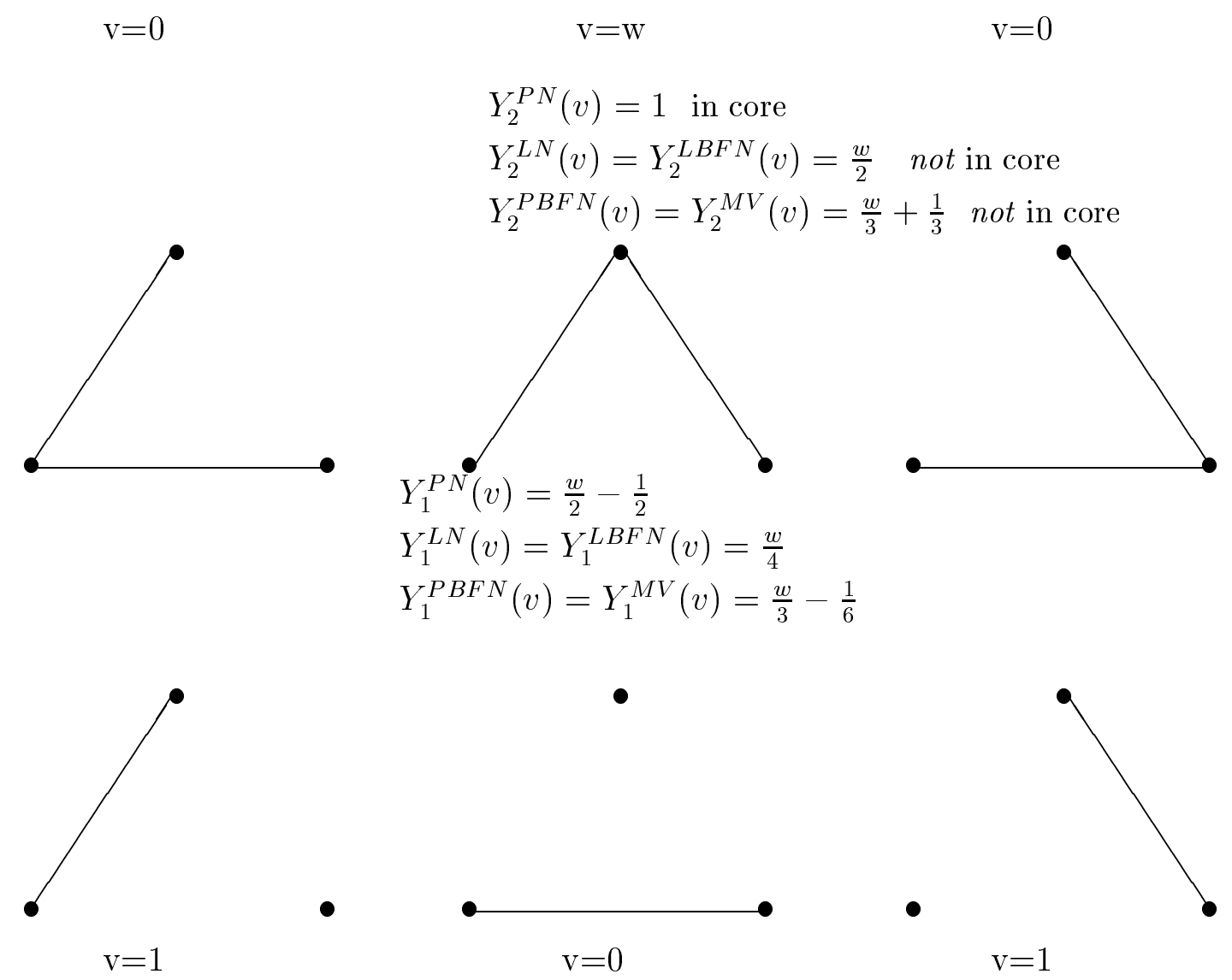

Figure 5

The failure of Shapley Value style calculations to turn up core allocations is wellknown from the cooperative game theory literature and it appears here too.

The flexible network approach that I have proposed here can also be combined with other ways of dividing up value among a group of players (or links). Thus, there is a natural variation of the Nucleolus that can be defined for network games.

The Nucleolus defined by Schmeidler (1969) has the very desirable property that it always lies in the core of a cooperative game whenever that object is non- empty, among some other nice properties. There is a natural analog of the nucleolus that can be defined from a flexible network perspective in network games. I will refer to that allocation rule as the networkolus. There are two versions, depending on whether the definition is linkbased or player-based.

I will begin with the player-based version as that always lies in the core of a network game when it is non-empty. 


\section{The Player-Based Networkolus}

Let $B(g, v)=\left\{y \in \mathbb{R}^{n} \mid \sum_{i} y_{i}=v(g)\right\}$ be the balanced allocations for $g$ under $v$.

Let $e_{S}(y)=\sum_{i \in S} y_{i}-\widehat{v}\left(g^{S}\right)$ be the excess allocated to coalition $S$ relative to their monotonic value under $v$, and let $e(y)$ denote the vector with entries for each nonempty $S \subset N$.

Let $Y^{P N}\left(g^{N}, \widehat{v}\right)=y$ be the unique allocation such that $e(y)$ leximin dominates $e\left(y^{\prime}\right)$ for all $y^{\prime} \in B\left(g^{N}, \widehat{v}\right)$. Then define $Y^{P N}(g, v)=\frac{v(g)}{\widehat{v}(g)} Y^{P N}\left(g^{N}, \widehat{v}\right)$.

We can also consider a link-based version of the networkolus.

\section{The Link-Based Networkolus}

Let $B^{\ell}(g, v)=\left\{x \in \mathbb{R}^{n(n-1) / 2} \mid \sum_{i} x_{i}=v(g)\right\}$ be the balanced allocations to links for $g$ under $v$.

Let $e_{g}^{\ell}(x)=\sum_{i j \in g} x_{i j}-\widehat{v}(g)$ be the excess allocated to set of links $g$ relative to their monotonic value under $v$, and let $e^{\ell}(x)$ denote the vector with entries for each nonempty $g \subset g \subset N$.

Let $Y_{i}^{L N}\left(g^{N}, \widehat{v}\right)=\sum_{j \neq i} y_{i j}$ be the unique allocation such that $e^{\ell}(y)$ leximin dominates $e^{\ell}(x)$ for all $x \in B^{\ell}\left(g^{N}, \widehat{v}\right)$. Then define $Y^{L N}(g, v)=\frac{v(g)}{v(g)} Y^{L N}\left(g^{N}, \widehat{v}\right)$.

Example 10 The Networkolus and the Core

Consider the value function $v$ from Example 9 defined by $v(\{12\})=v(\{23\})=1$, $v(\{12,23\})=w$, and $v(g)=0$ for all other networks.

Recall that the Myerson Value, Link-Based Flexible Network Bargaining Allocation Rule and the Player-Based Flexible Network Bargaining Allocation Rule failed to be core consistent in this example.

Let us examine the networkolus in this example, and consider the case where $3 \geq$ $w \geq 1 .{ }^{12}$

$Y^{P N}(\{12,23\}, v)=\left(\frac{w-1}{2}, 1, \frac{w-1}{2}\right)$ which indeed is in the core.

Note however, that the link-based version of the networkolus is not in the core. In this particular example it actually coincides with $Y^{L B F N}$. That is,

\footnotetext{
${ }^{12}$ The same conclusions hold in other cases, but involve different calculations.
} 
$Y^{L N}(\{12,23\}, v)=\left(\frac{w}{4}, \frac{w}{2}, \frac{w}{4}\right)$ which is not in the core.

See Figure 5.

The difficulty with the link-based networkolus is that it is making allocations based on links rather than players, while it is the players who are critical in core calculations. This actually suggests a refinement of the core, which is a link-based core.

\section{The Link-Core of a Network Game}

A network-allocation pair $g \subset g^{N}$ and $y \in \mathbb{R}^{n(n-1) / 2}$ is in the link-core of the network game $(N, v)$ if $\sum_{i j} y_{i j} \leq v(g)$ and $\sum_{i j \in g^{\prime}} y_{i j} \geq \widehat{v}\left(g^{\prime}\right)$ for all $g^{\prime} \subset g^{N}$.

The link-core of a network game provides a refinement of the core where it is links that are being allocated value. When the link-core is nonempty, it is straightforward to see that the core is nonempty. ${ }^{13}$ However, there are many situations (such as under the $v$ in Example 10) where the link-core is empty.

An allocation rule $Y$ is link-core consistent, if for any $v$ such that the link-core is nonempty, there exists at least one $g$ and $y \in \mathbb{R}^{n(n-1) / 2}$ such that $(g, y)$ is in the link-core and $\sum_{j \neq i} y_{i j}=Y_{i}(g, v)$ for each $i$.

Interestingly, the link-based networkolus is link-core consistent but not core consistent, while the player-based networkolus is core consistent but not link-core consistent.

Although I have not provided a characterization of the networkolus, there should be a straightforward variation on Peleg's (1986) reduced game property to derive a characterization of the networkolus that is an analog of that for the nucleolus.

\section{A General Approach to Importing Allocation Rules}

As should be clear by now, there is a general approach to defining allocation rules for network games that take a flexible-network perspective. For instance, one might want to define per-capita versions of the networkolus.

In general, let $\phi$ be your favorite allocation rule (a.k.a. imputation rule, solution, or value operator) from cooperative game theory. Noting that $\widehat{v}\left(g^{S}\right)$ defines a characteristic function as we vary $S$, we can apply $\phi$ directly and denote the resulting allocations by $\phi(\widehat{v})$.

To define a player-based rule, set $Y^{\phi}(g, v)=\frac{v(g)}{\widehat{v}(g)} \phi(\widehat{v})$.

\footnotetext{
${ }^{13}$ Let $g, \widehat{y}$ be in the link-core. Let $y \in \mathbb{R}^{n}$ be defined by $y_{i}=\sum_{j \neq i} \widehat{y}_{i j}$. Then $(g, y)$ lies in the core.
} 
To define a link-based rule, we can apply $\phi$ to groups of links rather than players (so on $\widehat{v}(g)$, viewing $g$ as a coalition of links) and denote the resulting allocations to links by $\phi^{\ell}$. Next, set $Y_{i}^{\phi^{\ell}}(g, v)=\frac{v(g)}{\widehat{v}(g)} \frac{1}{2} \sum_{j} \phi_{i j}^{\ell}(\widehat{v})$.

\section{Properties and Comparisons}

Let us consider some other properties that are of interest, and then make some comparisons across allocation rules.

\section{Anonymity}

Given a permutation of players $\pi$ (a bijection from $N$ to $N$ ) and any $g \in G$, let $g^{\pi}=\{\pi(i) \pi(j) \mid i j \in g\}$. Thus, $g^{\pi}$ is a network that shares the same architecture as $g$ but with the specific players permuted.

Given a permutation $\pi$, let $v^{\pi}$ be defined by $v^{\pi}(g)=v\left(g^{\pi^{-1}}\right)$ for each $g \in G$.

An allocation rule $Y$ is anonymous if for any $v, g \in G$, and permutation $\pi, Y_{\pi(i)}\left(g^{\pi}, v^{\pi}\right)=$ $Y_{i}(g, v)$.

Anonymity of an allocation rule requires that if all that has changed is the labels of the players and the value generated by networks has changed in an exactly corresponding fashion, then the allocation only change according to the relabeling. Of course, anonymity is a type of fairness condition that has a rich axiomatic history, and also naturally arises in situations where $Y$ represents the utility or productive value coming directly from some social network.

Note that anonymity allows for asymmetries in the ways that allocation rules operate even in completely symmetric networks. For instance, anonymity does not require that each player in a complete network get the same allocation. That would be true only in the case where $v$ was in fact anonymous. Generally, an allocation rule can respond to different roles or powers of players and still be anonymous.

Most allocation rules that one can think of satisfy this version of anonymity.

\section{Equal Treatment of Equals}

Given any two players $i$ and $j$ define a permutation $\pi^{i j}$ such that $\pi^{i j}(i)=j$ and $\pi^{i j}(j)=i$ and $\pi(k)=k$ for all $k \notin\{i, j\}$. Say that $i$ and $j$ are equals under $v$ if $v\left(g^{\pi^{i j}}\right)=v(g)$ for all $g$.

An allocation rule $Y$ satisfies equal treatment of equals if for any $v \in V$, players $i$ and $j$ who are equals under $v$, and $g \in G$ such that $g^{\pi^{i j}}=g, Y_{j}(g, v)=Y_{i}(g, v)$. 
Equal treatment of equals says that all allocation rule should give the same payoff to players who play exactly the same role in terms of symmetric position in a network under a value function that depends on them in exactly the same way. ${ }^{14}$

Equal treatment of equals is implied by anonymity and might be thought of as more of a symmetry condition than anonymity, and is also a condition that has a rich background in the axiomatic literature that will be satisfied by most allocation rules.

\section{Strong Anonymity}

A value function $v$ is anonymous if $v\left(g^{\pi}\right)=v(g)$ for any $g \in G$ and permutation $\pi$.

Anonymous value functions are those for which the architecture of a network matters, but not the labels of players.

An allocation rule $Y$ is strongly anonymous if $Y_{j}(g, v)=Y_{i}(g, v)$ for any anonymous $v, g \in G$, and players $i$ and $j$.

Strong anonymity states that if $v$ is anonymous, then the allocation be independent of players' positions in the network.

If the allocation is agreed upon at the same time (or before) the network is being formed, then the strong version of anonymity makes sense. From an ex ante point of view, when a given network $g$ is formed and the value function is anonymous, then any permutation of $g$ would have resulted in the same value and so in a sense the particular positions of the players become irrelevant. While players' ex post positions in the network may be asymmetric, their flexible network potential roles and contributions are completely identical.

Note that strong anonymity is in conflict with component balance. Note also that it implies that the allocation is completely egalitarian whenever $v$ is anonymous.

Before comparing a few allocation rules in terms of some of the conditions discussed here, a let us recall two other allocation rules. These were both defined by Jackson and Wolinsky (1996).

The Egalitarian Allocation Rule The egalitarian allocation rule $Y^{e}$ is defined by

$$
Y_{i}^{E}(g, v)=\frac{v(g)}{n}
$$

\footnotetext{
${ }^{14}$ There are various versions of this property. One might slightly strengthen the condition to require that $Y_{j}\left(g^{\pi^{i j}}, v\right)=Y_{i}(g, v)$ for any $g$. This condition would also be satisfied by almost any natural allocation rule.
} 
for all $i$ and $g$.

The egalitarian allocation rule splits the value of a network equally among all members of a society regardless of what their role in the network is. It is clear that the egalitarian allocation rule will have very nice properties in terms of aligning player incentives with efficiency.

\section{The Component-Wise Egalitarian Allocation Rule}

The egalitarian rule violates component balance. The following modification of the egalitarian rule respects component balance.

The component-wise egalitarian allocation rule $Y^{c e}$ is defined as follows for component additive $v$ 's and any $g$.

$$
Y_{i}^{C E}(g, v)= \begin{cases}\frac{v(h)}{|N(h)|} & \text { if there exists } h \in C(g) \text { such that } i \in h \\ 0 & \text { otherwise. }\end{cases}
$$

For any $v$ that is not component additive, set $Y^{c e}(\cdot, v)=Y^{e}(\cdot, v)$.

The component-wise egalitarian splits the value of a component network equally among all members of that component, but makes no transfers across components.

The component-wise egalitarian rule has some nice properties in terms of aligning player incentives with efficiency, although not quite to the extent that the egalitarian rule does. ${ }^{15}$

\footnotetext{
${ }^{15}$ See Jackson and Wolinsky (1996) Section 4 for some detailed analysis of the properties of the egalitarian and component-wise egalitarian rules.
} 


\begin{tabular}{|c|c|c|c|c|c|c|c|}
\hline & $Y^{M V}$ & $Y^{L B F N}$ & $Y^{L B F N}$ & $Y^{L N}$ & $Y^{P N}$ & $Y^{E}$ & $Y^{C E}$ \\
\hline anonymity & + & + & + & + & + & + & + \\
\hline $\begin{array}{l}\text { strong } \\
\text { anonymity }\end{array}$ & - & + & + & + & + & + & - \\
\hline $\begin{array}{l}\text { equal treatment } \\
\text { of vital links }\end{array}$ & - & + & - & + & - & - & - \\
\hline $\begin{array}{l}\text { equal treatment } \\
\text { of vital players }\end{array}$ & + & - & + & - & + & - & - \\
\hline additivity & + & - & - & - & - & + & + \\
\hline $\begin{array}{l}\text { weak } \\
\text { additivity }\end{array}$ & + & + & + & - & - & + & + \\
\hline flexible & - & + & + & + & + & + & - \\
\hline $\begin{array}{l}\text { network } \\
\text { proportional }\end{array}$ & - & + & + & + & + & + & - \\
\hline $\begin{array}{l}\text { equal bargaining } \\
\text { power }\end{array}$ & + & - & - & - & - & + & - \\
\hline $\begin{array}{l}\text { component } \\
\text { balance }\end{array}$ & + & - & - & - & - & - & + \\
\hline $\begin{array}{l}\text { core } \\
\text { consistency }\end{array}$ & - & - & - & - & + & - & - \\
\hline $\begin{array}{l}\text { link-core } \\
\text { consistency }\end{array}$ & - & - & - & + & + & - & - \\
\hline
\end{tabular}

\section{Endogenously Arising Allocation Rules}

Just as in cooperative game theory, providing an axiomatic foundation for an allocation rule leaves one agnostic on where allocation rules come from. From a normative perspective, these might be rules that we wish to impose, or that agents would naturally be led to by their own volition. It might also be that these naturally arise from some noncooperative bargaining procedure. For instance, one sees solutions such as the Shapley Value or the Nash Bargaining Solution popping up as the outcome or limit of outcomes of a variety of simple bargaining games.

Here one can also find such allocations arising as the outcomes of various noncooperative network formation and bargaining models. Mutuswami and Winter (2000) provide a non-cooperative game for network formation and the allocation of value, and it results in a Shapley Value-based allocation that differs from the Myerson Value. ${ }^{16}$ In

\footnotetext{
${ }^{16}$ See also, for instance, Navarro and Perea (2002) as well as Perez-Castrillo and Wettstein (2001), which provide for an implementation of the Myerson and Shapley Values, respectively, in different settings.
} 
fact, one can see that the allocation that Mutuswami and Winter obtain is the PlayerBased Flexible Network Allocation on the class of games that they consider. It would be interesting to further understand the connections between allocation rules and the possible network bargaining games that lead to them. 


\section{References}

Aumann, R. and Myerson, R. (1988) "Endogenous Formation of Links Between Players and Coalitions: An Application of the Shapley Value," In: Roth, A. (ed.) The Shapley Value, Cambridge University Press, 175-191.

Borm, P., Owen, G., and S. Tijs, S. (1992) "On the Position Value for Communication Situations," SIAM Journal on Discrete Mathematics, 5, 305-320.

Currarini, S. (2002) "Stable Organizations with Externalities," mimeo: Universitá di Venizia.

Currarini, S. and Morelli, M. (2000) "Network Formation with Sequential Demands," Review of Economic Design, 5, 229-250.

Dutta, B. and Mutuswami, S. (1997) "Stable Networks," Journal of Economic Theory, 76, 322-344.

Goyal, S. (2003) "Learning in Networks," forthcoming in Group Formation in Economics: Networks, Clubs, and Coalitions, edited by G. Demange and M. Wooders, Cambridge University Press: Cambridge.

Jackson, M. O. (2003). "A Survey of the Formation of Networks: Stability and Efficiency," forthcoming in Group Formation in Economics: Networks, Clubs, and Coalitions, edited by G. Demange and M. Wooders, Cambridge University Press: Cambridge.

Jackson, M. O. and Wolinsky, A. (1996) "A Strategic Model of Social and Economic Networks," Journal of Economic Theory, 71, 44-74.

Meeson, R. (1988) "Communication games," Master's thesis, Department of Mathematics, University of Nijmegen.

Mutuswami, S. and Winter, E. (2000) "Subscription Mechanisms for Network Formation," mimeo: CORE and Hebrew University in Jerusalem.

Myerson, R (1977) "Graphs and Cooperation in Games," Math. Operations Research, vol. 2, pp. 225-229.

Navarro, N. and A. Perea (2001) "Bargaining in Networks and the Myerson Value," mimeo: Universidad Carlos III de Madrid.

Page, F. (2003) "Farsighted Stability in Network formation," forthcoming in Group Formation in Economics: Networks, Clubs, and Coalitions, edited by G. Demange and M. Wooders, Cambridge University Press: Cambridge.

Peleg, B. (1986) "On the Reduced Game Property and its Converse," International Journal of Game Theory, 15, 187-200. 
Perez-Castrillo, D. and D. Wettstein (2001) "Bidding for the Surplus: A NonCooperative Approach to the Shapley Value," Journal of Economic Theory, 100, 274-294.

Schmeidler, D. (1969) "The Nucleolus of a characteristic function game," SIAM Journal of Applied Mathematics, 17, 1163-1170.

Slikker, M. (2000) Decision Making and Cooperation Structures CentER Dissertation Series: Tilburg.

van den Nouweland, A. (2003) "Static Networks and Coalition Formation," forthcoming in Group Formation in Economics: Networks, Clubs, and Coalitions, edited by G. Demange and M. Wooders, Cambridge University Press: Cambridge.

Wang, P. and Wen, Q. (1998) "Network Bargaining," mimeo: Penn State University.

Watts, A. (2001) "A Dynamic Model of Network Formation," Games and Economic Behavior, 34, 331-341. 


\section{Appendix}

I prove Theorem 7, as the proofs of Theorems 5 and 6 are straightforward analogs of this proof.

Proof of Theorem 7: It is easily checked that $Y^{L B F N}$ satisfies equal treatment of vital links and is an flexible network (and proportional) rule. Let us show that it satisfies weak additivity.

Consider any monotonic $v$ and $v^{\prime}$, and scalars $a \geq 0$ and $b \geq 0$. Then $a v+b v^{\prime}$ is monotonic and the same as its monotonic cover. So,

$$
\begin{gathered}
Y_{i}^{L B F N}\left(g^{N}, a v+b v^{\prime}\right) \\
=\sum_{j \neq i}\left[\sum_{g \subset g^{N}-i j} \frac{1}{2}\left(a v(g+i j)+b v^{\prime}(g+i j)-a v(g)-b v^{\prime}(g)\right)\left(\frac{\# g !([n(n-1) / 2]-\# g-1) !}{[n(n-1) / 2] !}\right)\right] .
\end{gathered}
$$

Given the monotonicity of $v$ and $v^{\prime}$, the right hand side can be rewritten as $a Y_{i}^{L B F N}\left(g^{N}, v\right)+$ $b Y_{i}^{L B F N}\left(g^{N}, v^{\prime}\right)$ which is the desired conclusion. Next, suppose that $a v-b v^{\prime}$ is monotonic. Then we can write

$$
\begin{gathered}
Y_{i}^{L B F N}\left(g^{N}, a v-b v^{\prime}\right) \\
=\sum_{j \neq i}\left[\sum_{g \subset g^{N}-i j} \frac{1}{2}\left(a v(g+i j)-b v^{\prime}(g+i j)-a v(g)+b v^{\prime}(g)\right)\left(\frac{\# g !([n(n-1) / 2]-\# g-1) !}{[n(n-1) / 2] !}\right)\right] .
\end{gathered}
$$

Given the monotonicity of $v$ and $v^{\prime}$, the right hand side can be rewritten as $a Y_{i}^{L B F N}\left(g^{N}, v\right)-$ $b Y_{i}^{L B F N}\left(g^{N}, v^{\prime}\right)$ which is again the desired conclusion.

Next, let us verify that any allocation rule satisfying equal treatment of vital links, weak additivity, and flexible network must coincide with $Y^{L B F N}$ on efficient networks. Proportionality then easily completes the second part of the theorem.

Consider an allocation rule satisfying the given properties. It is enough to show that it is uniquely determined on efficient networks.

The fact that $Y$ is a flexible network allocation rule implies that $Y(g, v)=Y\left(g^{N}, \widehat{v}\right)$ for an efficient network $g$, and so we need only show that $Y\left(g^{N}, \widehat{v}\right)$ is uniquely determined on an efficient network.

By the monotonicity of $\widehat{v}$, we can write

$$
\widehat{v}=\sum_{g^{\prime} \in G} c_{g^{\prime}} v_{g^{\prime}},
$$

for a set of scalars $c_{g^{\prime}}$ 's. 
Let $G^{-}=\left\{g^{\prime}: c_{g^{\prime}}<0\right\}$ and let $G^{+}=G \backslash G^{-}$. So we can write

$$
\widehat{v}=\sum_{g^{\prime} \in G^{+}} c_{g^{\prime}} v_{g^{\prime}}-\sum_{g^{\prime} \in G^{-}}\left|c_{g^{\prime}}\right| v_{g^{\prime}} .
$$

Thus, by weak additivity

$$
Y\left(g^{N}, \widehat{v}\right)=Y\left(g^{N}, \sum_{g^{\prime} \in G^{+}} c_{g^{\prime}} v_{g^{\prime}}\right)-Y\left(g^{N}, \sum_{g^{\prime} \in G^{-}}\left|c_{g^{\prime}}\right| v_{g^{\prime}}\right) .
$$

Applying weak additivity again allows us to write

$$
Y\left(g^{N}, \widehat{v}\right)=\sum_{g^{\prime}} c_{g^{\prime}} Y\left(g^{N}, v_{g^{\prime}}\right)
$$

Since $Y$ is a flexible network rule and both $g^{N}$ and $g^{\prime}$ are efficient for the monotonic $v_{g^{\prime}}$, $Y\left(g^{N}, v_{g^{\prime}}\right)=Y\left(g^{\prime}, v_{g^{\prime}}\right)$. By equal treatment of vital links, this (and thus the right hand side of the equation above) is uniquely determined. 